\title{
52. DOS NOVEDADES DEL GÉNERO TEUCRIUM SECCIÓN STACHYOBOTRYS BENTH. Y SECCION SCORODONIA (HILL) SCHREB. SUBSECCIÓN SCORODONIA KÄSTNER (LAMIACEAE) PARA LA FLORA EUROPEA.
}

Teresa NAVARRO y Baltasar CABEZUDO

Two news records of the Teucrium genus section Stachyobotrys Benth. and section Scorodonia (Hill) Schreb. subsection Scorodonia Kästner (Lamiaceae) for the Flora Europaea.

Palabras clave: Teucrium, Lamiaceae, Flora Europea.

Key words: Teucrium, Lamiaceae, Flora Europaea.

El género Teucrium L. ha sido estudiado en la Península Ibérica por numerosos autores (Navarro, 1995). La presencia en Andalucía de Teucrium bracteatum Desf. (Sect. Stachyobotrys Benth.) y T. afrum (Emb. \& Maire) Pau \& Font Quer subsp. afrum [Sect. Scorodonia (Hill.) Schreb. Subsect. Scorodonia Kästner], constituyen dos novedades para la flora europea. Es posible que T. afrum subsp. afrum haya sido ocasionalmente confundido con T. pseudoscorodonia Desf. [=T. scorodonia subsp. baeticum (Boiss. \& Reuter) Tutin)], con el que guarda cierta semejanza morfológica y con el que comparte el área de distribución.

Ambas especies han sido localizadas puntualmente en la provincía de Málaga, $T$. bracteatum en un encinar de Sierra Prieta sobre una ladera de gleras calcáreas de orientación sureste y T. afrum subsp. afrum en un encinarpinsapar de la Sierra de las Nieves sobre una ladera de orientación noreste y sustrato calizo. El aislamiento de estas poblaciones en relación a la distribución geográfica y variabilidad morfológica del material estudiado en el norte de África (Navarro, 1997), establece la hipótesis de su expansión a la Península desde el continente africano.

De todas las especies que integran el género Teucrium en la Península Ibérica e islas Baleares, sólo cinco son exclusivas de España y el norte de África. T. afrum y T. rotundifolium Schreb. (Sect. Polium Subsect. Rotundifolia Cohen ex Valdés Berm. \& Sánchez Crespo) del sur de España y Marruecos, T. bracteatum del sur-oeste de España, Marruecos y Algeria y $T$. pseudoscorodonia (Sect. Scorodonia Subsect. Scorodonia) y $T$. resupinatum Desf. (Sect. Spinularia Boiss.) del sur-oeste de España, Marruecos, Algeria y Túnez.

Con la presencia de $T$. bracteatum en España se amplía la distribución de la Sect. Stachyobotrys en el Mediterráneo occidental donde sólo está integrada por dos especies; $T$. bracteatum y $T$. collincola Greuter \& Burdet, este último endemismo marroquí.

El tratamiento sistemático específico seguido para $T$. afrum, es el propuesto por Castroviejo \& Bayón (1989) y para la clasificación infragenérica el propuesto por Navarro (op. cit.).

A continuación se relacionan los pliegos de herbario testigo de la cita de los dos nuevos taxones en la provincia de Málaga.

\section{Teucrium bracteatum Desf.}

Hs, MÁlAGA: Casarabonela, Sierra Prieta, 30SVF37, 800-1000m, 21-V-1997, Cabezudo \& Navarro. MGC 44953 . Ibidem, 6-VII-1993, Cabezudo, P. Navas, Y. Gil, Pérez-Latorre \& D. Navas, MGC 36236. 
Teucrium afrum (Emb. \& Maire) Pau \& Font Quer subsp. afrum

Hs, MÁLAGA: Sierra de Ronda, Parauta, 24VII-1987, Pérez-Latorre, MGC 22978.
Navarro, T. -1997-Sinopsis of the Teucrium genus in Morocco. (Lamiaceae). Acta Bot. Malacitana 22: $187-203$.

\section{BIBLIOGRAFÍA}

CASTROVIEJO, S y E. BAYÓN -1989.

Consideraciones sobre Teucrium Sect. Scorodonia (Hill) Schreber. Anales Jard. Bot. Madrid 42(2): 512-513.

NAVARRO, T. -1995- Revisón del género Teucrium Sect. Polium (Mill.) Schreb. en la Península Ibérica e Islas Baleares. Acta Bot. Malacitana 20: $173-265$.

Aceptado para su publicación en Octubre de 1997

Dirección de los autores. Dpto. Biología Vegetal. Facultad de Ciencias. Universidad de Málaga. Apdo. 59. 29080, Málaga. Fax: (95) 21319 44. E-mail: endemico@uma.es

\section{DISTRIBUTION AND ECOLOGY OF SOME RARE PLANT SPECIES IN NORTHERN MOROCCO I: TEUCRIUM AFRUM SSP. RUBRIFLORUM AND EUPHORBIA TRANSTAGANA.}

Ulrich DEIL

Zur Verbreitung und Ökologie seltener Pflanzen in Nordmarokko I. Teucrium afrum ssp. rubriflorum und Euphorbia transtagana.

Key words. Heathland, endemics, Morocco, Tingitanian sector.

Stichwörter. Heiden, Endemiten, Marokko, Tingitanischer Sektor.

In a series of papers, remarks will be made about the distribution and the ecology of some rare plants in Morocco. This first contribution deals with two species: Teucrium afrum ssp. rubriflorum and Euphorbia transtagana. The ecology of the species is described, their localities are mapped and their phytosociology is discussed by a number of relevés. Finally, the conservation value of the species and the vulnerability of their habitats will be discussed. The tax onomic nomenclature follows mainly Valdes et al. (1987), the syntaxonomical nomenclature Deil (1997). 